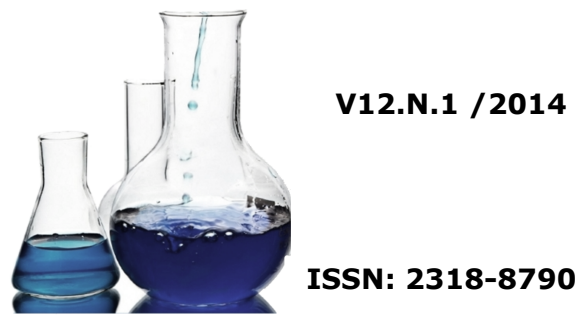

\title{
Equipe de Trabalho 2014
}

\section{Equipe editorial}

\section{Editor-Chefe}

- Bayardo Bapstista Torres, Instituto de Química - USP, Brasil

- Eduardo Galembeck, Departamento de Bioquímica Instituto de Biologia UNICAMP, Brasil

\section{Editores}

- Gabriel Gerber Hornink, Depto. Bioquímica, Instituto de Ciências Biomédicas, Universidade - Federal de Alfenas - Unifal-MG, Brasil

- Vera Maria Treis Trindade, Instituto de Ciências Básicas da Saúde, Departamento de Bioquímica, Universidade Federal do Rio Grande do Sul, Brasil

\section{Corpo Editorial}

- Adriana Cassina, Departamento de Bioquímica, Facultad de Medicina, Universidad de la República, Uruguai

- Angel Herráez, Departamento de Bioquímica y Biología molecular, Universidad de Alcalá de Henares, Madrid, Espanha

- André Amaral Gonçalves Bianco, Universidade Federal de São Paulo (Unifesp), Brasil

- Denise Vaz de Macedo, Departamento de Bioquímica, Instituto de Biologia, Universidade Estadual de Campinas - Unicamp, Brasil

- Eneida de Paula, Departamento de Bioquímica, Instituto de Biologia, Universidade Estadual de Campinas - Unicamp, Brasil

- Guilherme Andrade Marson, Instituto de Química - USP, Brasil

- Jose Antonio Martinez Oyanedel, Universidad de Concepción, Chile

- Josep Maria Fernández Novell, Dept. Bioquímica i Biologia Molecular Universitat de Barcelona, Espanha

- Leila Maria Beltramini, Instituto de Física de São Carlos, Universidade Estadual de São Paulo - USP, Brasil

- Manuel João da Costa, Escola de Ciências da Saúde, Universidade do Minho, Portugal

- Maria Lucia Bianconi, Instituto de Bioquímica Médica Universidade Federal do Rio de Janeiro (UFRJ), Brasil

- María Noel Alvarez, Departamento de Bioquímica, Facultad de Medicina, Universidad de la República, Uruguai

- Miguel Ángel Medina Torres, Department of Molecular Biology \& Biochemistry Faculty of Sciences University of Málaga, Espanha

- Nelma Regina Segnini Bossolan, Instituto de Física de São Carlos, Universidade de São Paulo - USP, Brasil

- Paulo De Avila Junior, Centro de Ciências Naturais e Humanas (CCNH) Universidade Federal do ABC (UFABC), Brasil

- Raul Herrera Faúndez, Instituto de Biología Vegetal y Biotecnologia, Universidad de Talca, Chile

- Wagner Seixas da Silva, Instituto de Bioquímica Médica, Universidade Federal do Rio de Janeiro (UFRJ), Brasil 


\section{Avaliadores (Jan-Jul, 2014)}

- Adriana Cassina, Departamento de Bioquímica, Facultad de Medicina, Universidad de la República, Uruguai

- André Amaral Gonçalves Bianco, Universidade Federal de São Paulo (Unifesp), Brasil

- Daniela Kiyoko Yokaichiya, Doutora em Bioquímica (Unicamp).

- Eduardo Galembeck, Departamento de Bioquímica Instituto de Biologia, UNICAMP, Brasil

- Gabriel Gerber Hornink, Depto. Bioquímica, Instituto de Ciências Biomédicas, Universidade Federal de Alfenas (Unifal-MG), Brasil

- Jair Adriano Kopke de Aguiar, Departamento de Bioquímica/ICB, Universidade Federal de Juiz de Fora (UFJF), Brasil

- José Maurício Scheedorf da Silva, Departamento de Bioquímica/ ICB, Universidade Federal de Alfenas (Unifal-MG)

- Leila Maria Beltramini, Instituto de Física de São Carlos, Universidade Estadual de São Paulo - USP, Brasil

- Manuel João da Costa, Escola de Ciências da Saúde, Universidade do Minho, Portugal

- Maria Elena Infante-Malachias, Universidade de São Paulo (USP), Brasil

- Maria Lucia Bianconi, Instituto de Bioquímica Médica Universidade Federal do Rio de Janeiro (UFRJ), Brasil

- Mauricio Roberto Motta Pinto da Luz, Instituto Oswaldo Cruz - Fundação Oswaldo Cruz

- Nelma Regina Segnini Bossolan, Instituto de Física de São Carlos, Universidade de São Paulo - USP, Brasil

- Paulo De Avila Junior, Centro de Ciências Naturais e Humanas (CCNH) Universidade Federal do ABC (UFABC), Brasil

- Paulo Granjeiro, Laboratório de Processos Biotecnológicos e Purificação de Macromoléculas, Universidade Federal de São João Del-Rei (UFJS), Brasil

- Vera Maria Treis Trindade, Instituto de Ciências Básicas da Saúde, Departamento de Bioquímica, Universidade Federal do Rio Grande do Sul (UFRGS), Brasil

- Wagner Seixas da Silva, Instituto de Bioquímica Médica, Universidade Federal do Rio de Janeiro (UFRJ), Brasil

\section{Apoio institucional}

- SBBq - Sociedade Brasileira de Bioquímica e Biologia Molecular

$\circ$ http://www.sbbq.org.br

- Presidente em exercício: Moacir Wajner

- Vice-presidente: Carlos Termignoni

- Secretário geral: Walter R. Terra

- Primeiro Secretário: Sandro Roberto Marana

- Tesoureira geral: Alícia Juliana Kowaltowiski

- Primeiro tesoureiro: Maurício da Silva Baptista

- Coordenador de Política Internacional: Anibal Eugenio Vercesi

- Coordenador de Relações Internacional: Richard Garrat 